\title{
Performance of the CMS Jets and Missing Transverse Energy Trigger at LHC Run 2
}

\author{
Jane Nachtman ${ }^{1}$ \\ University of Iowa \\ Iowa City, Iowa, USA \\ E-mail: jane-nachtman@uiowa.edu \\ Ismail Okan Atakisi \\ Bogazici University \\ Istanbul, Turkey \\ E-mail: atakisi.okan@cern.ch \\ Milos Dordevic \\ University of Belgrade \\ Belgrade, Serbia \\ E-mail:milos.djordjevic@cern.ch

\section{Mithat Kaya} \\ Bogazici University \\ Istanbul, Turkey \\ E-mail: mithat.kaya@cern.ch
}

\section{Ozlem Kaya}

Bogazici University

Istanbul, Turkey

E-mail:ozlem.kaya@cern.ch

\section{Henning Kirschenmann}

CERN

Geneva, Switzerland

E-mail: henning.kirschenmann@cern.ch

\section{Fengwangdong Zhang}

Universite Libre de Bruxelles

Brussels, Belgium

E-mail: fengwangdong.zhang@cern.ch

\footnotetext{
${ }^{1}$ Speaker, supported by the U.S. Department of Energy Office of Science award DESC0010113.
} 
In preparation for collecting proton-proton collisions from the LHC at a center-of-mass energy of $13 \mathrm{TeV}$ and rate of $40 \mathrm{MHz}$ with increasing instantaneous luminosity, the CMS collaboration prepared an array of triggers utilizing jets and missing transverse energy for searches for new physics at the energy frontier as well as for SM precision measurements. The CMS trigger system must be able to sift through the collision events in order to extract events of interest at a rate of $1 \mathrm{kHz}$, applying sophisticated algorithms adapted for fast and effective operation. Particularly important is the calibration of the trigger objects, as corrections to the measured energy may be substantial. Equally important is the development of improved reconstruction algorithms to mitigate negative effects due to high numbers of overlapping proton-proton collisions and increased levels of beam-related effects. Work by the CMS collaboration on upgrading the high-level trigger for jets and missing transverse energy for the upgraded LHC operation will be presented, along with the improved performance of these triggers.

38th International Conference on High Energy Physics 3-10 August 2016

Chicago, USA 


\section{Jet reconstruction on high level trigger (HLT) and offline}

A consequence of the increase in the instantaneous luminosity and bunch crossing frequency is a large increase in the overlapping energy signatures in the detector from simultaneous interactions ("in-time pileup") and interactions from the previous bunch crossing ("out-of-time pileup"). This results in a large contribution of artificial jets which are not from the hard-scatter vertices.

Figure 1 shows the ratio between number of pileup jets and number of all reconstructed jets for three different reconstruction scenarios. While the RunI hadronic energy reconstruction simply integrates the signal in a certain time window, the RunII hadronic energy reconstruction (both online and offline) separates "in-time" and "out-of-time" pulses. The RunII reconstruction is superior in suppressing pileup jets.
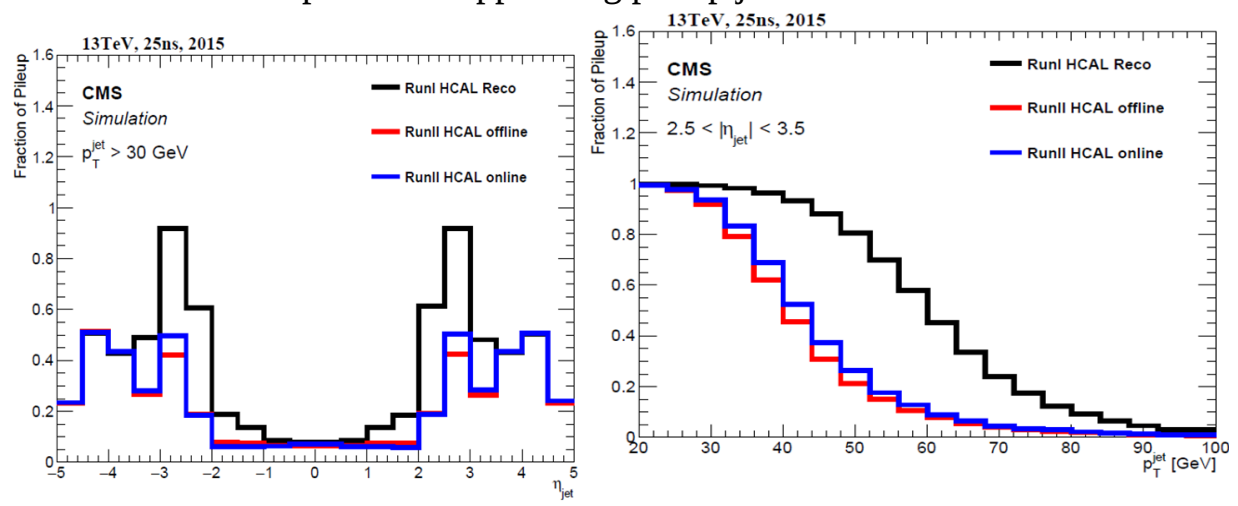

Figure 1 : Pileup jets fraction

with different reconstruction

scenarios, showing the increase in

pileup jets at Run 2 and the

effective mitigation.

\section{Jet energy correction and calibration}

Reconstructed jets at CMS are corrected using Jet Energy Corrections (JEC), determined from simulation. These corrections are done in three stages: Level 1 (L1) is the pileup correction, Level 2 (L2) is the relative eta correction, and Level 3 (L3) is the absolute transverse momentum correction. The L1 Pileup correction is derived from a simulation of single-neutrino events with multiple interactions per event. In bins of eta we take cones that are randomly chosen in phi, with the size of our jets (cone in eta-phi space with radius up to 0.4), and sum the energy from the three different types of Particle Flow particles: Photons, Charged Hadrons, Neutral Hadrons. We can then derive an average contribution from Pileup as a function of eta and the number of interactions, as shown in Figure 2. 

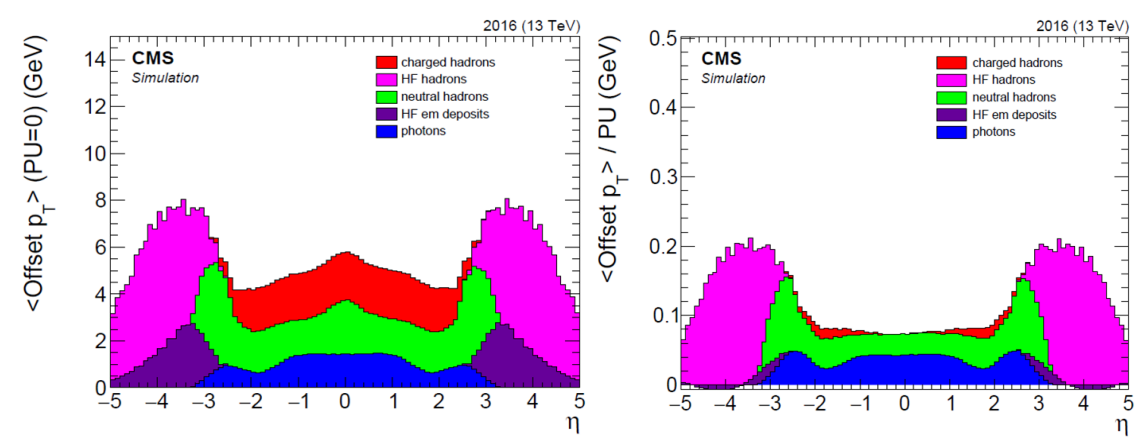

Figure 2: Random-Cone Average Offset transverse momentum measured in Monte Carlo simulations per True Pileup as a function of eta in the HLT reconstruction, showing the various particle type contributions, in simulated data with a flat distribution of 0 to 50 Pileup events. The left plot shows the offset extrapolated to 0 pileup events, and the right plot shows the offset /pileup.

The Jet Energy Corrections are applied at the HLT to two types of jets: Calorimeter jets, which use only the calorimeter information, and Particle Flow jets, which use calorimeter and tracking information. The performance of the Jet Energy Corrections are compared in Figure 3 for both types of jets.
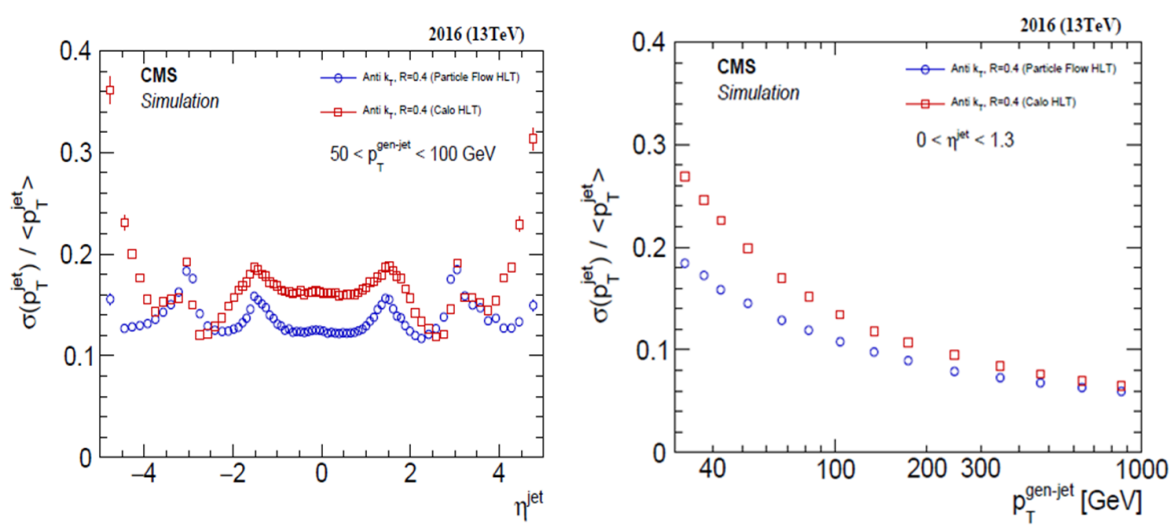

Figure 3: Jet energy resolution as a function of the reconstructed jet eta (left) and of the generated jet (gen-jet) transverse momentum (right), after applying the jet energy corrections in the High Level Trigger.

\section{Application of JEC to Missing Transverse Energy}

Missing Transverse Energy (MET), or transverse energy imbalance, is an important event quantity used in searches for new physics We have improved the performance of the MET 
triggers by applying Jet Energy Corrections to the jets used in the MET calculation, as shown in
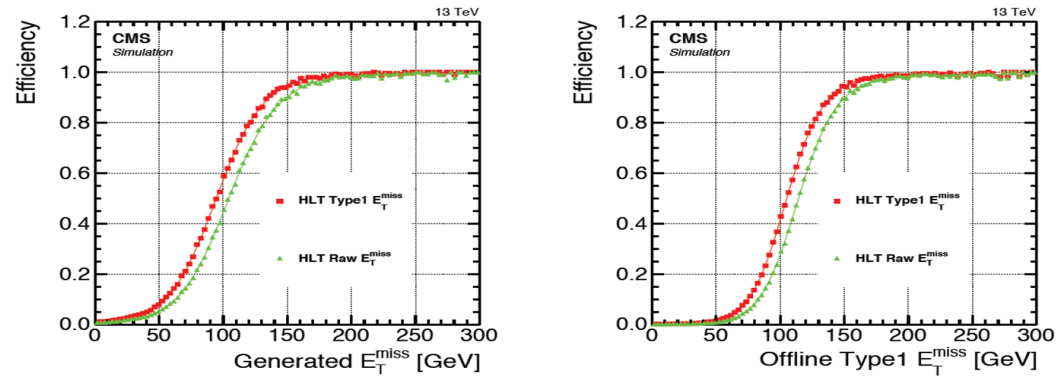

Figure 4: Efficiency of MET trigger with a threshold at 150

$\mathrm{GeV}$. The 3-4\% improvement in the trigger turn-on curve allows

a higher threshold to be used at HLT, lowering the rate while giving the same performance [1].

Figure 4.

\section{Conclusions}

The CMS High Level Triggers for Jets and Missing Transverse Energy are continually being updated to cope with increasing instantaneous luminosity as the LHC continues to deliver oustanding performance. Applying more sophisticated corrections and algorithms at the trigger level allows CMS to produce high-quality physics results at the energy frontier.

\section{References}

[1] CMS Collaboration, Performance of the Missing Energy Reconstruction in $13 \mathrm{TeV}$ pp data, CMSJME-16-004. 